\title{
Contractions of Representations of de Sitter Groups
}

\author{
J. MICKELSSON ${ }^{\star}$ and J. NIEDERLE ${ }^{\star \star}$ \\ International Atomic Energy Agency, and United Nations Educational Scientific and \\ Cultural Organization International Centre for Theoretical Physics
}

Received January 17, 1972

\begin{abstract}
In order to construct the quantum field theory in a curved space with no "old" infinities as the curvature tends to zero, the problem of contraction of representations of the corresponding group of motions is studied. The definitions of contraction of a local group and of its representations are given in a coordinate-free manner. The contraction of the principal continuous series of the de Sitter groups $S O_{0}(n, 1)$ to positive mass representations of both the Euclidean and Poincaré groups is carried out in detail. It is shown that all positive mass continuous unitary irreducible representations of the resulting groups can be obtained by this method. For the Poincare groups the contraction procedure yields reducible representations which decompose into two non-equivalent irreducible representations.
\end{abstract}

\section{Introduction}

It was stressed several times by Salam [1] that divergent integrals in quantum field theory may be replaced by regularized ones using Lagrangians intrinsically non-polynomial in character, in particular, all gravity - modified matter Lagrangians. The gravity constant $\kappa$ then plays the role of a cut-off (provided there are no other constants in the theory than $\left.\frac{1}{\kappa} \sim 10^{19} \mathrm{GeV}\right)$. Thus, we may hope that by taking gravity into account, we can make the quantum field theory free of infinities.

We shall try to check this idea in another way, without assuming the quantization of gravity but with the Einstein geometrical interpretation of gravity instead. In other words, we shall construct the quantum field theory in a curved space the curvature of which will hopefully play the role of cut-off. Following Fronsdal [2] we may even hope that the quantum field theory in a curved space in some well-defined limit goes not only to the usual field theory in Minkowski flat space - time but also

* Institute of Theoretical Physics, Göteborg, Sweden.

$\star \star$ On leave of absence from the Institute of Physics of the Czechoslovak Academy of Sciences. Prague, Czechoslovakia.

12 Commun math Phys., Vol. 27 
to a new one with no "old" infinities as the curvature tends to zero. It means that the class of physical theories obtainable as limits as the curvature tends to zero may be more restrictive.

In this paper we begin the programme and study the limiting procedure by which the representations of the de Sitter group (the group of motions of space with constant curvature) go to the representations of the Poincare group (the group of motions of the Minkowski space). This procedure, called according to Wigner and Inönü [3] contraction, is also suitable for elucidating the relation between external and internal symmetries, for the unification of different theories, for finding representations of contracted Lie groups by knowing those of the initial groups, etc., etc. That is the reason we study a more general case - contractions of the principal continuous series of representations of the de Sitter groups $S O_{0}(n, 1)^{1}$ to all non-zero mass representations of both the Poincare group $E_{0}(n-1,1)^{2}$ and the Euclidean group $E(n)$.

The process of contraction has, by now, an extensive literature. The first contraction process was proposed by Segal [4] and the more specific ones by Inönü and Wigner [3], Saletan [5], by Doebner and Melsheimer [6] and by Zajcev (see the review article by Lykhmus [7] which also contains other references).

In most of the papers the authors propose different contractions of Lie algebras rather than of the Lie groups. Besides differences, the approaches have also a common feature arising from the following wellknown facts. The Lie algebra of a given Lie group considered as a linear space is described by basis elements (generators of the algebra) together with the commutation relations among them. As shown by Cartan, whenever we change the basis by a non-singular transformation the resulting basis will describe the generators of an algebra isomorphic to the original one. However, if the transformation is singular, a new algebra may be received, provided that the properties of the commutator to be the commutator of a Lie algebra are fulfilled. The papers [3-7] indicate different ways of performing this singular transformation and necessary and sufficient conditions that a given Lie algebra can be contracted into another one. In practice, we consider a sequence of transformations of basis elements rather than one transformation. Transformations in the sequence depend on one (or more) parameter $\varepsilon$ such that for all values of parameter $\varepsilon$ except one the corresponding transformation is regular and in the particular point (usually $\varepsilon=0$ ) the transformation becomes singular. We may divide different approaches into two categories depending whether

${ }^{1} \mathrm{SO}_{0}(n, 1)$ denotes the identity component of the pseudo-orthogonal group $O(n, 1)$.

${ }^{2} E_{0}(n, 1)$ denotes the identity component of the Poincare group with $n$ spatial and 1 timelike dimension. 
i) all generators that depend on $\varepsilon$ tend to zero in the same way as $\varepsilon \rightarrow 0$ or;

ii) whether some generators vanish faster then the others as $\varepsilon \rightarrow 0$.

The typical simple examples of both categories can be obtained considering first the Lie algebra of $S U(2)$, i.e., the algebra of angular momentum, with commutation relations

$$
\left[J_{1}, J_{2}\right]=J_{3}, \quad\left[J_{2}, J_{3}\right]=J_{1}, \quad\left[J_{3}, J_{1}\right]=J_{2}
$$

and taking the transformation of generators either

i) $J_{i} \rightarrow J_{i}^{c}(\varepsilon)=\varepsilon J_{i}, i=1,2, J_{3} \rightarrow J_{3}^{c}(\varepsilon)=J_{3}$ or

ii) $J_{i} \rightarrow J_{i}^{c}(\varepsilon)=\varepsilon J_{i}, i=1,2, J_{3} \rightarrow J_{3}^{c}(\varepsilon)=\varepsilon^{2} J_{3}$.

As $\varepsilon \rightarrow 0$ we get new algebras - the algebra of the Euclidean group $E(2)=S O(2) \otimes T_{2}$ with the commutation relations

$$
\left[J_{1}^{c}, J_{2}^{c}\right]=0, \quad\left[J_{2}^{c}, J_{3}^{c}\right]=J_{1}^{c}, \quad\left[J_{3}^{c}, J_{1}^{c}\right]=J_{2}^{c}
$$

and the Heisenberg algebra with the commutation relations

$$
\left[J_{1}^{c}, J_{2}^{c}\right]=J_{3}^{c}, \quad\left[J_{2}^{c}, J_{3}^{c}\right]=\left[J_{1}^{c}, J_{3}^{c}\right]=0 \quad \text { respectively. }
$$

From these examples we can easily see which generators previously not commuting do commute as $\varepsilon \rightarrow 0$ as well as why contractions [3-7] are not satisfactory from the point of view of representation theory. Really, in contracting representations we find the following main difficulties:

i) contracting the faithful representation of a given Lie algebra we obtain, in general, a non-faithful representation of the resulting Lie algebra (all generators multiplied by $\varepsilon$ go to zero operators as $\varepsilon \rightarrow 0^{3}$ ).

ii) The resulting (contracted) Lie algebra is always non-compact. Hence any contraction of a Hermitian (continuous) irreducible representation of some compact Lie algebra, which is always finite-dimensional, has to yield at the end an infinite-dimensional Hermitian irreducible representation of the non-compact Lie algebra. This difficulty is not removed even if we start with a representation of the non-compact Lie algebra which has already an infinite-dimensional representation space because the contraction is coupled with a modification of the representation space (see [8]).

Wigner and Inönu in [3] (see also [8]) mentioned two ways of treating the difficulties. In the first, they suggest using a similarity transformation, $A_{\varepsilon} \cdot J_{i}^{c}(\varepsilon) \cdot A_{\varepsilon}^{-1}$, which does not change commutation relations, but, if it depends on $\varepsilon$ in a tricky way ${ }^{3}$, can make representations of all

${ }^{3}$ In this section we clarify different points by using the simplest contraction procedure [3], however, the statements are also true for other procedures [5-7] which depend on more parameters $\varepsilon$ and their powers as well. 
generators non-trivial as $\varepsilon \rightarrow 0$. In the second, they change one representation of the initial algebra to another simultaneously with the change of $\varepsilon$. Thus, a sequence of representations rather than some particular representation goes to one contracted representation. In other words, as $\varepsilon$ decreases, the dimensionalities of the representations in the sequence increase so that the resulting representation space becomes infinitedimensional as $\varepsilon \rightarrow 0$.

These methods were applied for contractions of representations of the following "de Sitter" groups: $S O_{0}(2,1)$ into $E_{0}(1,1)$ in [8], $S O_{0}(4,1)$ into $E_{0}(3,1)$ in [9], $S O_{0}(3,2)$ into $E_{0}(3,1)$ in $[10]$ and $S O_{0}(n, 1)$ into $E(n)$ in [11]. However, no attempt was made at full generality nor at complete mathematical rigor in these papers. With the exception of [8], the authors used only infinitesimal approaches (i.e., contracted representations of algebras) which give rise to two delicate problems in addition the question of domains of definitions of generators ${ }^{4}$ and the question of integrability of representations.

In order to avoid these complications we study the problem of contractions of representations first of all globally, i.e., for the Lie groups. We try to formulate the problem also more rigorously and generally. Thus, in Section II we give the definitions of the contraction of a local group and of its representations as well. The introduced definition of the contraction of a Lie group differs essentially from that of Saletan [5] since it is given in a coordinate-free manner. Sections III and IV are devoted to the contraction of the principal continuous series of the de Sitter groups $S O_{0}(n, 1)$ to all positive mass representations of the Euclidean group $E(n)$ and of the Poincare group $E_{0}(n-1,1)$, respectively. Roughly speaking, it is shown that all continuous unitary irreducible representations with positive mass of the group $E(n)$ as well as $E_{0}(n-1,1)$ can be obtained by the method. This result for $E(n)$ agrees with conclusions made by Wolf in [12]. For $E_{0}(n-1,1)$, the contraction procedure yields reducible representations which decompose into two non-equivalent irreducible representations.

\section{Definition of Contraction of Groups and of Their Representations}

Saletan [5] has given a definition of the contraction of a Lie group by using a one-parameter family of coordinate transformations. However, this definition is not convenient in many cases, because, in general, it is not always possible to cover the whole group by only one set of coordinates. Of course, if we want to work only in some neighbourhood of the identity, then one set of coordinate is sufficient.

${ }^{4}$ In a continuous Hermitian representation of the non-compact Lie algebra some generators are unbounded. 
We shall give here a global, coordinate-free definition, of the contraction of a topological group $G$ to a topological group $G^{c}$. In fact, we shall only assume that $G$ is defined locally.

Definition 1. Let $G$ be a local group [13] and $G^{c}$ a topological group. We say that $G^{c}$ is a contraction of $G$ if there exists a neighbourhood $V$ of the identity e in $G$ such that $V^{2}=\{x y \mid x, y \in V\}$ is defined and a family of mappings

such that

$$
f_{\varepsilon}: V^{2} \rightarrow G^{c} ; \quad \varepsilon \in[1,0)
$$

i) $f_{\varepsilon}$ is a homeomorphism from $V^{2}$ onto $f_{\varepsilon}\left(V^{2}\right)$ for $\forall \varepsilon \in[1,0)$,

ii) if $x^{c} \in G^{c}$ then $\exists \varepsilon_{0} \in[1,0)$ such that $x^{c} \in f_{\varepsilon}(V)$ if $\varepsilon<\varepsilon_{0}$, i.e., $f_{\varepsilon}^{-1}\left(x^{c}\right)$ is defined and belongs to $V$ when $\varepsilon<\varepsilon_{0}$,

iii) $f_{\varepsilon}(e)$ is the identity in $G^{c}$ for $\forall \varepsilon \in[1,0)$,

iv) if $x, y \in G^{c}$ then $x \cdot y=\lim _{\varepsilon \rightarrow 0} f_{\varepsilon}\left(f_{\varepsilon}^{-1}(x) \cdot f_{\varepsilon}^{-1}(y)\right)$.

We illustrate this definition by several examples.

Example 1. Let $G$ be any topological group and $G^{c}$ the identity component of $G$. Let $V=G^{c}$ and $f_{\varepsilon}$ be the identity map on $V=V^{2}$. It is easily seen that $f_{\varepsilon}$ satisfies all the conditions of Definition 1.

Example 2. Let $G=S^{1}$ (the additive group of a circle), parametrized in the form $e^{i \varphi},-\pi \leqq \varphi<\pi$ and $G^{c}=R^{1}$ (the additive group of a real line). Let $V=\{\varphi \mid \varphi \in(-1,1)\}$, so that $V^{2}=\{\varphi \mid \varphi \in(-2,2)\}$, and let $f_{\varepsilon}: V^{2} \rightarrow R^{1}$ be defined as $f_{\varepsilon}(\varphi)=\frac{1}{\varepsilon} \varphi$. It is easy to prove again that conditions i)-iv) of Definition 1 are fulfilled.

Example 3. Let $G=S O_{0}(n, 1)$ and $G^{c}=E(n)$. Every element of $G$ can be written uniquely in the form $g=k \cdot p$, where $k \in S O(n)$ and

$$
p=\exp \left\{i \sum_{j=1}^{n} t_{j} M_{j, n+1}\right\},
$$

where $M_{j, n+1}$ is the non-compact generator of a hyperbolic rotation in the $(j, n+1)$-plane. This is the Cartan decomposition for $G$ [14]. Topologically $p \sim R^{n}$. For $g \in G, g=k \cdot p\left(t_{1}, \ldots, t_{n}\right)$ where $k$ can be represented by an $(n \times n)$ orthogonal matrix with matrix elements $k_{\mu \nu}$. Every element of $G^{c}$ can be written in the form $k \cdot r\left(t_{1}, \ldots, t_{n}\right), k \in S O(n)$ and $r\left(t_{1}, \ldots, t_{n}\right)$ is a translation by the vector $t=\left(t_{1}, \ldots, t_{n}\right)$. Let now $V=V^{2}=G$ and $f_{\varepsilon}(k \cdot p(t))=k \cdot r\left(\frac{1}{\varepsilon} t\right)$. The conditions i)-iii) are obviously satisfied. The 
condition iv) can be checked as follows:

$$
\begin{aligned}
\lim _{\varepsilon \rightarrow 0} & f_{\varepsilon}\left(f_{\varepsilon}^{-1}\left(k^{(1)} \cdot r\left(t^{(1)}\right)\right) \cdot f_{\varepsilon}^{-1}\left(k^{(2)} \cdot r\left(t^{(2)}\right)\right)\right) \\
& =\lim _{\varepsilon \rightarrow 0} f_{\varepsilon}\left(k^{(1)} \cdot p\left(\varepsilon t^{(1)}\right) \cdot k^{(2)} \cdot p\left(\varepsilon t^{(2)}\right)\right) \\
& =\lim _{\varepsilon \rightarrow 0} f_{\varepsilon}\left(k^{(1)} \cdot k^{(2)} \cdot\left(k^{(2)}\right)^{-1} \cdot p\left(\varepsilon t^{(1)}\right) \cdot k^{(2)} \cdot p\left(\varepsilon t^{(2)}\right)\right) .
\end{aligned}
$$

If $K$ denotes the Lie algebra of $S O(n)$ and $P=\left\{M_{j, n+1}, j=1,2, \ldots, n\right\}$, then $[K, P] \subset P$ and $[P, P] \subset K$. Therefore, to the first order in $\varepsilon$ we have

$$
\begin{aligned}
\left(k^{(2)}\right)^{-1} \cdot p\left(\varepsilon t^{(1)}\right) \cdot k^{(2)} \cdot p\left(\varepsilon t^{(2)}\right) & \approx p\left(\varepsilon k_{j i}^{(2)} t_{j}^{(1)}\right) \cdot p\left(\varepsilon t^{(2)}\right) \\
& \approx p\left(\varepsilon k_{j i}^{(2)} t_{j}^{(1)}+\varepsilon t_{i}^{(2)}\right) .
\end{aligned}
$$

where $\varepsilon k_{j i}^{(2)} t_{j}^{(1)}$ means the vector $\left\{\varepsilon k_{j 1}^{(2)} t_{j}^{(1)}, \varepsilon k_{j 2}^{(2)} t_{j}^{(1)}, \ldots, \varepsilon k_{j n}^{(2)} t_{j}^{(1)}\right\}$. Combining Eqs. (2.1) and (2.2) we obtain

$$
\lim f_{\varepsilon}\left(f_{\varepsilon}^{-1}\left(k^{(1)} \cdot r\left(t^{(1)}\right)\right) \cdot f_{\varepsilon}^{-1}\left(k^{(2)} \cdot r\left(t^{(2)}\right)\right)\right)=k^{(1)} \cdot k^{(2)} \cdot r\left(k_{j i}^{(2)} t_{j}+t_{i}^{(2)}\right)
$$

which is equal to $k^{(1)} \cdot r\left(t^{(1)}\right) \cdot k^{(2)} \cdot r\left(t^{(2)}\right)$.

Example 4. $G=S O_{0}(n, m)$ and $G^{c}=E_{0}(n, m-1)$.

Let $A^{\prime}=\left\{p \mid p \in G, p=\exp \left[i \sum_{j=1}^{n+m-1} t_{j} M_{j, n+m}\right]\right\}$. Here, $M_{j, n+m}$ is the noncompact generator of a hyperbolic rotation in the $(j, n+m)$-plane. Let $V^{\prime}=\left\{g \mid g \in G, g=k \cdot p, k \in S 0_{0}(n, m-1), p \in A^{\prime}\right\}$. Then there exists $\Delta>0$ such that if $V=\left\{g \mid g \in V^{\prime}, g=k \cdot p(t),-\Delta<t_{j}<\Delta, j=1,2, \ldots, n\right\} \subset V^{\prime}$ then $V^{2} \subset V^{\prime}$. As in the previous example, we put $f_{\varepsilon}(k \cdot p(t))=k \cdot r\left(\frac{1}{\varepsilon} t\right)$, where $r(t)$ is again a translation by the vector $t$. Using the same arguments as before, we can show that $E_{0}(n, m-1)$ is a contraction of $S_{0}(n, m)$.

The contractions of the groups in Examples 2-4 correspond to the usual Inönü-Wigner contractions of the corresponding Lie algebras.

Next we want to define what is meant by the contraction of a representation. For this purpose, we consider a one-parameter family of representations $\mathscr{D}^{\varepsilon}(g)$ of $G$ in Hilbert spaces $\mathscr{H}^{\varepsilon}$, i.e., $\left\{\mathscr{D}^{\varepsilon}(g)\right.$ in $\left.\mathscr{H}^{\varepsilon}, \varepsilon \in[1,0)\right\}$. We assume that representations $\mathscr{D}^{\varepsilon}$ are continuous and unitary. Let $A_{\varepsilon}$ be a continuous linear mapping from $\mathscr{H}^{\varepsilon}$ to a Hilbert space $\mathscr{H}$ such that $A_{\varepsilon}: \mathscr{H}^{\varepsilon} \rightarrow A_{\varepsilon}\left(\mathscr{H}^{\varepsilon}\right)$ is unitary and for $\forall \psi \in \mathscr{H}$ there exists $\varepsilon_{\psi}$ such that $\varepsilon<\varepsilon_{\psi} \Rightarrow \psi \in A_{\varepsilon}\left(\mathscr{H}^{\varepsilon}\right)$.

Definition 2. With the same notation as in Definition 1 if the limit of $A_{\varepsilon} \mathscr{D}^{\varepsilon}\left(f_{\varepsilon}^{-1}(g)\right) A_{\varepsilon}^{-1} \psi$ exists for $\forall \psi \in \mathscr{H}, \forall g \in G^{c}$ as $\varepsilon \rightarrow 0$ and is continuous in $g$, and the homomorphism $G^{c} \ni g \rightarrow \mathscr{D}(g)$ defined by

$$
\mathscr{D}(g) \psi=\lim _{\varepsilon \rightarrow 0} A_{\varepsilon} \mathscr{D}^{\varepsilon}\left(f_{\varepsilon}^{-1}(g)\right) A_{\varepsilon}^{-1} \psi
$$

is a unitary representation of $G^{c}$, the representation $g \rightarrow \mathscr{D}(g)$ of $G^{c}$ is said to be a contraction of the representation $g \rightarrow \mathscr{D}^{1}(g)$ of $G$. 


\section{Contraction of Principal Series of Representations of $S O_{0}(n, 1)$ to All Non-Zero Mass Representations of $E(n)$}

Let $G=K A N$ be the Iwasawa decomposition for $G=S O_{0}(n, 1)[14]$. Let $M$ be the centralizer of $A$ in $K, M=S O(n-1)$. We denote by $(l, s)$ the irreducible representation of $P=M A N$ in the vector space $\mathscr{H}^{(l)}$, defined by

$$
P \ni \operatorname{man} \rightarrow \mathscr{D}^{l}(m) \cdot e^{s t}
$$

where $m \in M, a \in A, n \in N$ and $\mathscr{D}^{l}$ is the $U I R$ of $M$ characterized by the highest weight $l=\left(l_{1}, \ldots, l_{\left[\frac{1}{2}(n-1)\right]}\right)$ and $t$ is a parameter for the elements in $A$. The representation of $G$ induced by the representation $(l, s)$ of $P$ and by the $K$-invariant measure $d \mu(x)$ on $X=G / P \approx S^{n-1}$ will be denoted $U^{(l, s)}$. If $s=\frac{n-1}{2}+i v, v$ real, $v \neq 0$, it will be unitary and irreducible [15].

The Hilbert space in which $U^{(l, s)}$ acts is the space $L_{2}^{(l)}(X)$ of all square integrable functions on $X$ with values in the vector space $\mathscr{H}^{(l)}$. The scalar product is defined by

$$
(\varphi, \psi)=\int_{X} \overline{\varphi(x)} \cdot \psi(x) d \mu(x) .
$$

The action of $U^{(l, s)}(g)$, the representative of $g \in G$, is given by

$$
\left(U^{(l, s)}(g) \varphi\right)(x)=e^{s t(g, x)} \mathscr{D}^{l}\left(m\left(k_{g x}^{-1} g k_{x}\right)\right) \varphi(g \cdot x),
$$

where $x \in X$ is presented by a $n$-component vector with unit length, $x^{2}=1, k_{x}$ is any element of $S O(n)$ such that $k_{x}(1,0, \ldots, 0)=x, t(g, x)$ $=t\left(g k_{x}\right) ; g k_{x}=k a(t) n$ and $m\left(k_{g x}^{-1} g k_{x}\right)$ means the first factor in the decomposition $k_{q x}^{-1} g k_{x}=\operatorname{man}, m \in M, a \in A$ and $n \in N$. As the generator of $a(t)$ we will take $M_{1, n+1}$ (see Example 3, Section II). Now, we will put $G^{c}=E(n)$ and the family of maps $\left\{f_{\varepsilon}\right\}$ as in Example 3 in Section II. The linear mapping $A_{\varepsilon}$ in Definition 2 is taken as the identity map 1 for every $\varepsilon \in[1,0)$. Moreover, we put $s=\frac{n-1}{2}+i \frac{\varrho}{\varepsilon}$. Let us consider the limit

$$
\begin{aligned}
\lim _{\varepsilon \rightarrow 0} & A_{\varepsilon} U^{(l, s)}\left(f_{\varepsilon}^{-1}(g)\right) A_{\varepsilon}^{-1} \varphi(x) \\
= & \lim _{\varepsilon \rightarrow 0} \exp \left[\left(\frac{n-1}{2}+\frac{i \varrho}{\varepsilon}\right) \cdot t\left(f_{\varepsilon}^{-1}(g), x\right)\right] \\
& \cdot \mathscr{D}^{l}\left(m\left(k_{f^{-1}}^{-1}(g) x \cdot f_{\varepsilon}^{-1}(g) \cdot k_{x}\right)\right) \cdot \varphi\left(f_{\varepsilon}^{-1}(g) \cdot x\right),
\end{aligned}
$$

where $\varphi(x) \in L_{2}^{(l)}(X)$ and $g \in G^{c}$. If $g=k \in S O(n)$ then we notice that for every $\varepsilon$ the expression above is equal to

$$
\mathscr{D}^{l}\left(m\left(k_{k x}^{-1} \cdot k \cdot k_{x}\right)\right) \varphi(k \cdot x)
$$

and so is the limit $\varepsilon \rightarrow 0$. 
Assume, now, that $g=r(p)$, i.e., a translation by the $n$-vector $p=\left(p_{1}, \ldots, p_{n}\right)$. Then,

$$
f_{\varepsilon}^{-1}(r(p))=\exp \left[i \varepsilon \sum_{j=1}^{n} p_{j} M_{j, n+1}\right] .
$$

After putting (3.3) into (3.1) we obtain for the limit

$$
\lim _{\varepsilon \rightarrow 0} \exp \left[\left(\frac{n-1}{2}+i \frac{\varrho}{\varepsilon}\right) \cdot t\left(f_{\varepsilon}^{-1}(r(p), x)\right)\right] \varphi(x) .
$$

On the other hand,

$$
\begin{aligned}
t\left(f_{\varepsilon}^{-1}(r(p), x)\right) & =t\left(f_{\varepsilon}^{-1}(r(p)) \cdot k_{x}\right)=t\left(k_{x}^{-1} \cdot f_{\varepsilon}^{-1}(r(p)) \cdot k_{x}\right) \\
& =t\left\{\exp \left[i \varepsilon \sum_{l, j=1}^{n}\left(\left(k_{x}^{-1}\right)_{j l} \cdot p_{l} \cdot M_{j, n+1}\right)\right]\right\}+0\left(\varepsilon^{2}\right) \\
& =\sum_{i} \varepsilon\left(k_{x}^{-1}\right)_{1 i} p_{i}
\end{aligned}
$$

Combining (3.4) and (3.5) we get for the limit, which we denote by $\mathscr{D}^{\mathfrak{Q}, l}(r(p)) \varphi(x)$ :

$$
\mathscr{D}^{\varrho, l}(r(p)) \varphi(x)=e^{i \varrho x \cdot p} \varphi(x) .
$$

Similarly, we denote the limit in (3.2) by

$$
\mathscr{D}^{\varrho, l}(k) \varphi(x)=\mathscr{D}^{l}\left(m\left(k_{k x}^{-1} \cdot k \cdot k_{x}\right)\right) \varphi(k \cdot x) .
$$

Eqs. (3.6) and (3.6') define the $U I R$ of $E(n)$ characterized by the representation $\mathscr{D}^{l}$ of the little group $S O(n-1)$ and by mass squared $=\varrho^{2}$. But up to this we have only shown that at every point $x \in X$

$$
\lim _{\varepsilon \rightarrow 0} A_{\varepsilon} U^{(l, s)}\left(f_{\varepsilon}^{-1}(g)\right) A_{\varepsilon}^{-1} \varphi(x)=\mathscr{D}^{\varrho, l}(g) \varphi(x), \quad g \in G^{c} .
$$

Next, we want to prove that

$$
\lim _{\varepsilon \rightarrow 0}\left\|A_{\varepsilon} U^{(l, s)}\left(f_{\varepsilon}^{-1}(g)\right) A_{\varepsilon}^{-1} \varphi-\mathscr{D}^{\varrho, l}(g) \varphi\right\|=0, \quad \varphi \in L_{2}^{(l)}(x),
$$

which is the condition appearing in Definition 2. Consider the subspace $S \subset L_{2}^{(l)}(X)$ consisting of all continuous functions on $X$ with values in $\mathscr{H}^{(l)}$. $S$ is dense in $L_{2}^{(l)}(X)$. Since $X$ is compact, any element of $S$ is a bounded function on $X$. Let $\varphi \in S$ be fixed,

We define

$$
\|\varphi(x)\|_{\mathscr{H}(l)}<M \quad \forall x \in X .
$$

$$
F_{\varepsilon}(x)=\left\|A_{\varepsilon} U^{(l, s)}\left(f_{\varepsilon}^{-1}(g)\right) A_{\varepsilon}^{-1} \varphi(x)-\mathscr{D}^{\varrho, l}(g) \varphi(x)\right\|_{\mathscr{H}(l)}^{2} .
$$


Using (3.1) and (3.5) it is easily seen that there exists $M^{\prime}>0, \varepsilon_{0}>0$ such that $F_{\varepsilon}(x)<M^{\prime} \forall x \in X, \varepsilon<\varepsilon_{0}$. Because of (3.7)

$$
\lim _{\varepsilon \rightarrow 0} F_{\varepsilon}(x)=0 .
$$

The left-hand side of (3.8) is equal to (when $\varphi \in S$ )

$$
\lim _{\varepsilon \rightarrow 0}\left[\int F_{\varepsilon}(x) d \mu(x)\right]^{\frac{1}{2}}=\left[\int \lim _{\varepsilon \rightarrow 0} F_{\varepsilon}(x) d \mu(x)\right]^{\frac{1}{2}}=0 .
$$

$X$ is of finite measure and the functions $F_{\varepsilon}(x)$ are uniformly bounded when $\varepsilon<\varepsilon_{0}$, thus the order of integration and $\lim _{\varepsilon \rightarrow 0}$ can be interchanged.

As $S$ is dense in $L_{2}^{(l)}$ and all operators under consideration are unitary, the condition $\varphi \in S$ in (3.10) can be dropped and (3.8) follows.

Thus, we have proved the following theorem:

Theorem 1. Every UIR of E(n) characterized by "mass-squared" $=\varrho^{2}>0$ and by the representation $\mathscr{D}^{l}, l=\left(l_{1}, l_{2}, \ldots, l_{\left[\frac{1}{2}(n-1)\right]}\right)$, of the little group $S O(n-1)$, can be contracted from the representation $U^{(l, s)}$, $s=\frac{n-1}{2}+i \varrho$, of the group $S O_{0}(n, 1)$.

\section{Contraction of Principal Series of Representations of $S O_{0}(n, 1)$ to Positive Mass-Squared Representations of $E_{0}(n-1,1)$}

Let us denote by $B$ the connected component of the identity of the subgroup of $S_{0}(n, 1)$ which leaves invariant the first component of any vector in the defining representation. $B=S O_{0}(n-1,1) . B^{\prime}$ is the normalizer of $B$ in $G=S O_{0}(n, 1) ; B^{\prime}=B \cup B \tau$, where

$$
\tau=\operatorname{diag}(-1,-1, \underbrace{1,1, \ldots, 1}_{(n-1) \text { tumes }}) .
$$

We should like first to transform the representation $U^{(l, s)}$ of $G$ to a new basis which makes the reduction $G \downarrow B$ explicit (for further details see [16] where $n=2,3,4$ and [17] for arbitrary $n$, however, ladder representations; the general case is studied in [18]).

According to [18] almost every element of $G$ can be written in the form

$$
g=b a n ; \quad g \in G, \quad b \in B^{\prime}, \quad a \in A, \quad n \in N .
$$

Because of this decomposition, the representation $U^{(l, s)}$ of $G$ can be realized also in the space $L_{2}^{(l)}(\tilde{X})$ of square-integrable functions on the manifold $\tilde{X}=B^{\prime} A N / M A N=B^{\prime} / M$ instead of the manifold $X=K A N / M A N=K / M$. The action of $G$ in $L_{2}^{(l)}(\tilde{X})$ is given by [18]

$$
U^{(l, s)}(g) \varphi(x)=e^{s t(g, x)} \mathscr{D}^{l}\left(m\left(b_{g}^{-1} \cdot x \cdot g \cdot b_{x}\right)\right) \varphi(g \cdot x),
$$


where $\varphi(x)$ is a square-integrable function on $\tilde{X}$ with values in $\mathscr{H}^{(l)}, b_{x}$ is the coset representative of $x$ and $t(g, x)=t\left(g b_{x}\right)$. The scalar product is given by

$$
(\varphi, \psi)=\int_{x \in \hat{X}} \overline{\varphi(x)} \cdot \psi(x) d \mu(x)
$$

where $d \mu(x)$ is the $B^{\prime}$-invariant measure on $\tilde{X}$.

$\tilde{X}$ can be realized as a two-sheeted hyperboloid in the Euclidean space $R^{n}: x=\left(x_{1}, x_{2}, \ldots, x_{n}\right), x_{1}^{2}+x_{2}^{2}+\cdots+x_{n-1}^{2}-x_{n}^{2}=-1$. Then, $b_{x} \in B^{\prime}$ is the element which takes the vector $u=(0,0, \ldots, 0,1)$ to the vector $x$

$$
x_{i}= \pm \sum_{j=1}^{n}\left(b_{x}\right)_{i+1, j+1} u_{j}= \pm\left(b_{x}\right)_{i+1, n+1} .
$$

$b_{x}$ is a $(n+1) \times(n+1)$ matrix of the type

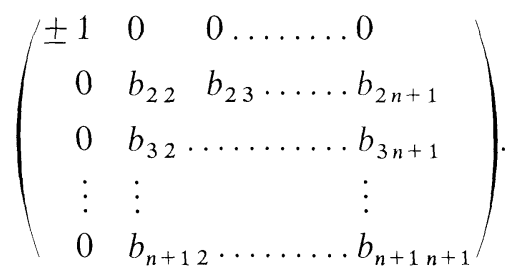

Let us note that under the subgroup $B \subset B^{\prime}$ the hyperboloids

$$
\tilde{X}^{ \pm}=\left\{x \mid x \in \tilde{X} \text { and } x_{n} /\left|x_{n}\right|= \pm 1\right\}
$$

remain invariant.

Now, we should like to contract the representations $U^{(l, s)}$ of $G$ to representations of the Poincare group in $n$-dimensions, $G^{c}=E_{0}(n-1,1)$. We use the family of mappings explained in Example 4, Section II. We set again $A_{\varepsilon}=1$ for all $\varepsilon \in[1,0)$. Let us denote by $g \rightarrow \mathscr{D}^{\varrho, l}(g)$ the representation of $G^{c}$ obtained. The contraction does not affect the action of the subgroup $B \subset G, B \subset G^{c}$ :

$$
\begin{aligned}
\mathscr{D}^{\varrho, l}(b) \varphi(x)=U^{(l, s)}(b) \varphi(x) & =\mathscr{D}^{l}\left(m\left(b_{b x}^{-1} \cdot b \cdot b_{x}\right)\right) \varphi(b \cdot x), \\
s & =\frac{n-1}{2}+i \varrho, \quad b \in B .
\end{aligned}
$$

Let us consider a translation $r(p) \in G^{c}$ by vector $p$.

Again, $\lim _{\varepsilon \rightarrow 0} f_{\varepsilon}^{-1}(r(p))=e$, the identity in $G$. Therefore,

$$
\begin{aligned}
\lim _{\varepsilon \rightarrow 0} U^{\left(l, \frac{n-1}{2}+i \frac{\varrho}{\varepsilon}\right)}\left(f_{\varepsilon}^{-1}(r(p))\right) \varphi(x) \\
\quad=\lim _{\varepsilon \rightarrow 0} \exp \left[i \frac{\varrho}{\varepsilon} \cdot t\left(f_{\varepsilon}^{-1}(r(p)), x\right)\right] \varphi(x) \equiv \mathscr{D}^{\varrho, l}(r(p)) \varphi(x) .
\end{aligned}
$$


In a similar way as in Section III, we obtain

$$
\lim _{\varepsilon \rightarrow 0} t\left(f_{\varepsilon}^{-1}(r(p)), x\right)=\varepsilon p \cdot x .
$$

It follows that (in the sense of pointwise convergence)

$$
\mathscr{D}^{\varrho, l}(r(p)) \varphi(x)=e^{i \varrho p \cdot x} \varphi(x) .
$$

In order to show that

$$
\lim _{\varepsilon \rightarrow 0}\left\|U^{\left(l, \frac{n-1}{2}+i \frac{\varrho}{\varepsilon}\right)}\left(f_{\varepsilon}^{-1}(r(p))\right) \varphi-\mathscr{D}^{\varrho . l}(r(p)) \varphi\right\|=0,
$$

we consider dense subspace $S \subset L_{2}^{(l)}(\tilde{X})$ of continuous functions with compact support. Let $\varphi \in S$ and let us denote

$$
\varphi_{\varepsilon}(x)=U^{\left(l \cdot \frac{n-1}{2}+\imath \frac{\varrho}{\varepsilon}\right)}\left(f_{\varepsilon}^{-1}(r(p))\right) \varphi(x) .
$$

It is easy to see that the functions $\varphi_{\varepsilon}(x), \varepsilon \in\left[\varepsilon_{0}, 0\right)$ are continuous. $\varepsilon_{0}$ is some positive number such that $f_{\varepsilon}^{-1}(r(p))$ is defined when $\varepsilon \leqq \varepsilon_{0}$.

We define $f_{0}^{-1}(r(p))=\lim _{\varepsilon \rightarrow 0} f_{\varepsilon}^{-1}(r(p))=e$. Consider the continuous mapping $g$, defined by

$$
g:\left[0, \varepsilon_{0}\right] \times \tilde{X} \rightarrow \tilde{X}, \quad g(\varepsilon, x)=f_{\varepsilon}^{-1}(r(-p)) \cdot x .
$$

$A_{0}$ is the support of $\varphi(x)$. As $\left[0, \varepsilon_{0}\right] \times A_{0}$ is compact and $g$ is continuous, the image of $\left[0, \varepsilon_{0}\right] \times A_{0}$ in $\tilde{X}$ is also compact. We denote it by $A$. From $\varphi_{\varepsilon}(x) \propto \varphi\left(f_{\varepsilon}^{-1}(r(p)) \cdot x\right)$ it follows that each $\varphi_{\varepsilon}(x), \varepsilon \in\left[\varepsilon_{0}, 0\right)$ is zero outside the compact set $A$. We define now

$$
F_{\varepsilon}(x)=\left\|\varphi_{\varepsilon}(x)-\mathscr{D}^{Q, l}(r(p)) \varphi(x)\right\|_{\mathscr{\mathscr { C }}(l)}^{2} .
$$

The left-hand side of (4.4) is equal to

$$
\lim _{\varepsilon \rightarrow 0}\left[\int_{\varepsilon} F_{\varepsilon}(x) d \mu(x)\right]^{\frac{1}{2}}=\int_{A}\left[\lim _{\varepsilon \rightarrow 0} F_{\varepsilon}(x) d \mu(x)\right]^{\frac{1}{2}}=0 .
$$

According to what has been said above, $F_{\varepsilon}(x)$ is zero outside $A$ when $\varepsilon \leqq \varepsilon_{0}$ and thus the integration in (4.5) can be restricted to $A$.

With this we have completed the proof of (4.4). Thus, we have shown that the representation $U^{\left(l, \frac{n-1}{2}+i \varrho\right)}$ of $G$ can be contracted to the representation

$$
\begin{aligned}
B \ni b & \rightarrow \mathscr{D}^{(l, \varrho)}(b): \mathscr{D}^{(l, \varrho)}(b) \varphi(x)=\mathscr{D}^{l}\left(m\left(b_{b}^{-1} \cdot x \cdot b \cdot b_{x}\right)\right) \varphi(b \cdot x), \\
r(p) & \rightarrow \mathscr{D}^{(l, \varrho)}(r(p)): \mathscr{D}^{(l, \varrho)}(r(p)) \varphi(x)=e^{i \varrho p \cdot x} \varphi(x)
\end{aligned}
$$

of $G^{c}$ 
We should like to reduce this result to irreducible representations. Let us consider first the subspace of $L_{2}^{(l)}(\tilde{X})$ consisting of functions which are zero on $\tilde{X}^{-}$. We denote it by $L_{2}^{(l)}\left(\tilde{X}^{+}\right)$. It is easily seen that $L_{2}^{(l)}\left(\tilde{X}^{+}\right)$ is invariant under the action of $G^{c}$. Furthermore, this subspace carries the irreducible representation of $G^{c}$ induced by the representation $\mathscr{D}^{l}$ of little group $M$ corresponding to the orbit characterized by the "time-like" vector $(0,0, \ldots, 0, \varrho)$.

The complement of $L_{2}^{(l)}\left(\tilde{X}^{+}\right)$in $L_{2}^{(l)}(\tilde{X})$ is the subspace $L_{2}^{(l)}\left(\tilde{X}^{-}\right)$, consisting of functions which are zero on $\tilde{X}^{+}$. From (4.2) and from the definition of $\tau$, it follows that $b_{-x}=b_{x} \cdot \tau$. Thus, we can rewrite (4.6) in the form:

$$
\begin{aligned}
\mathscr{D}^{l}\left(m\left(b_{b}^{-1} \cdot b \cdot b \cdot b_{x}\right)\right) \varphi(b \cdot x) & =\mathscr{D}^{l}\left(m\left(\tau b_{b}^{-1} \cdot\left(-x \cdot b \cdot b_{-x} \tau\right)\right) \varphi(-b \cdot(-x))\right. \\
& =A \mathscr{D}^{l}\left(m\left(\tau b_{b}^{-1} \cdot b \cdot b_{x} \cdot \tau\right)\right) \cdot A^{-1} \varphi(b \cdot x), \\
e^{I g p x} \varphi(x) & =A e^{i(-\varrho) p \cdot x} A^{-1} \varphi(x),
\end{aligned}
$$

where $A$ is a linear mapping from $L_{2}^{(l)}\left(\tilde{X}^{+}\right)$on to $L_{2}^{(l)}\left(\tilde{X}^{-}\right)$given by $A \varphi(x)=\varphi(-x)$.

From (4.7) we conclude that the restriction of the representation (4.6) of $G^{c}$ to the subspace $L_{2}^{(l)}\left(\tilde{X}^{-}\right)$is equivalent (via the linear mapping $A$ ) to the following representation, realized in the space $L_{2}^{(l)}\left(\tilde{X}^{+}\right)$:

$$
\begin{gathered}
B \ni b \rightarrow \overline{\mathscr{D}}^{(l, \varrho)}(b): \overline{\mathscr{D}}^{(l, \varrho)}(b) \varphi(x)=\mathscr{D}^{l}\left(m\left(\tau b_{b \cdot x}^{-1} \cdot b \cdot b_{x} \tau\right)\right) \varphi(b \cdot x), \\
r(p) \rightarrow \overline{\mathscr{D}}^{(l, \varrho)}(r(p)): \overline{\mathscr{D}}^{(l, \varrho)}(r(p)) \varphi(x)=e^{i(-\varrho) p \cdot x} \varphi(x), \\
\varphi \in L_{2}^{(l)}(\tilde{X}) .
\end{gathered}
$$

The representation (4.8) is induced by the characters of the translation subgroup which belong to the orbit of $B$ with the characteristic "timelike vector" $(0, \ldots, 0,-\varrho)$ and by the representation

$$
M \ni m \rightarrow \overline{\mathscr{D}}^{l}(m)=\mathscr{D}^{l}(\tau m \tau)
$$

of the little group $M$. We have to distinguish two cases: whether the group $S O_{0}(n, 1)$ is with $n$ even or $n$ odd.

$$
\text { a) } n \text { is even }(n=2 k)
$$

The group $M=S O(n-1)$ is the subgroup of $G$ consisting of the matrices

$$
m \in M, m=\left(\begin{array}{ccccc}
1 & 0 & 0 & \ldots & 0 \\
0 & & & 0 \\
\vdots & & T & & \vdots \\
\vdots & & & & \vdots \\
0 & 0 & 0 & \ldots & 1
\end{array}\right), T \text { is an }(n-1) \times(n-1) \text { matrix. }
$$


Consider subgroup $S O(n-2) \subset M$ with $T$ of the form

$$
T=\left(\begin{array}{cccc}
1 & 0 & \ldots & 0 \\
0 & & \\
\vdots & & T^{\prime} \\
0 &
\end{array}\right) \quad T^{\prime} \text { is an }(n-2) \times(n-2) \text { matrix. }
$$

It is easily seen that

$$
m \in S O(n-2) \Rightarrow \tau m \tau=m .
$$

The restriction of $\overline{\mathscr{D}}^{l}$ to $S O(n-2)$ is, therefore, equivalent to the restriction of $\mathscr{D}^{l}$ to $S O(n-2)$. Now, every irreducible representation of $S O(2 k-1)$ is completely determined (up to an equivalence) by its restriction to the subgroup $S O(2 k-2)$. It follows that $\overline{\mathscr{D}}^{l}$ is equivalent to $\mathscr{D}^{l}$

$$
\text { b) } n \text { is odd }(n=2 k+1)
$$

It is not true that irreducible representations of $S O(2 k)$ are completely determined by their restriction to the subgroup $S O(2 k-1)$. Let $l=\left(l_{1}, l_{2}, \ldots, l_{k}\right),\left(l_{1} \geqq l_{2} \geqq \cdots \geqq\left|l_{k}\right|\right)$ be the highest weight of an $I R$ of $S O(2 k)$. Let $\bar{l}=\left(l_{1}, l_{2}, \ldots,-l_{k}\right)$. Then the restrictions of $\mathscr{D}^{l}$ and $\mathscr{D}^{\bar{l}}$ to the subgroup $S O(2 k-1)$ are equivalent. By studying the effect of $\tau$ on the Cartan subalgebra $\left\{H_{i} \mid i=1,2, \ldots, k\right\}$ one finds that

$$
\begin{aligned}
\tau H_{i} \tau & =H_{i}, \quad i=1,2, \ldots, k-1, \\
\tau H_{k} \tau & =-H_{k},
\end{aligned}
$$

where $H_{i}, i=1,2, \ldots, k-1$ belong to the algebra of $S O(2 k-1)$ and $H_{k}$ does not. From (4.9) we conclude that $\overline{\mathscr{D}}^{l}$ is equivalent to $\mathscr{D}^{\bar{l}}$. The results of the last section lead to the following theorem:

Theorem 2. The representation $U^{(l, s)}$ of $S O_{0}(n, 1), s=\frac{n-1}{2}+i \varrho$, can be contracted to the representation $\mathscr{D}^{(l, \varrho)}$ of $E_{0}(n-1,1) . \mathscr{D}^{(l, \varrho)}=\mathscr{D}_{+}^{(l, \varrho)}$ $\oplus \mathscr{D}_{-}^{(l, \varrho)}$, where $\mathscr{D}_{ \pm}^{(l, \varrho)}$ are irreducible representations. $\mathscr{D}_{ \pm}^{(l, \varrho)}$ is induced by the representation $p \rightarrow e^{ \pm i \varrho p_{n}}$ of the translation subgroup and by the representation $\mathscr{D}_{ \pm}^{l}$ of the corresponding little group $S O(n-1) . \mathscr{D}_{+}^{l}=\mathscr{D}^{l}$ and $\mathscr{D}_{-}^{l}=\mathscr{D}^{\bar{l}}$, where $l=\bar{l}$, if $n$ is even, and $\bar{l}=\left(l_{1}, l_{2}, \ldots,-\frac{l_{\frac{n-1}{2}}}{2}\right)$, if $n$ is odd.

Acknowledgments. One of the authors (J.N.) would like to thank Professors Abdus Salam and P. Budini, as well as IAEA and UNESCO, for hospitality kindly extended to him at the International Centre for Theoretical Physics in Trieste, where the work was completed. The second author (J.M.) wishes to thank Dr. L. Pekárek for his hospitality at the Institute of Physics of the Czechoslovak Academy of Sciences in Prague, where almost the whole work was carried out as well as NORDITA for its financial support. He is also grateful to Dr. S. Ström for interesting discussions. 


\section{References}

1. See, e.g., Salam, A.: Preprint ICTP, IC/71/108, Trieste 1971.

2. Fronsdal, C.: Rev. Mod. Phys. 37, 221 (1965).

3. Inönü, E., Wigner, E.P.: Proc. Nat. Acad. Sci. U.S. 39, 510 (1953).

4. Segal, I.E.: Duke Math. J. 18, 221 (1951).

5. Saletan, E.J.: J. Math. Phys. 2, 1 (1961).

6. Doebner, H. D., Melsheimer, O.: Nuovo Cimento 49A, 306 (1967).

7. Lykhmus, J.: Contractions of Lie Groups, at the Proceedings of the 1967 Summer School on Elementary Particle Physics (held at Otepää, Estonian SSR), Vol. 4. Tallin 1969.

8. Philips, T.O., Wigner,E.P.: De Sitter space and positive energy. In: Group Theory and Its Applications, ed. by E.M. Loebl. New York and London: Academic Press, p. $631,1968$.

9. Ström, S.: Ark. Fysik 30, 455 (1965) see also S.Ström, Ann. Inst. Henri Poincaré 13, 77 (1970).

10. Evans, N. T.: J. Math. Phys. 8, 170 (1967).

11. Chakrabarti, A.: J. Math. Phys. 9, 2087 (1968); see also Schwartz,F.: J. Math. Phys. 12, 131 (1971).

12. Wolf, K. B.: J. Math. Phys. 12, 197 (1971).

13. Pontryagin, L. S.: Topological Groups, p. 137, New York-London-Paris: Gordon and Breach, Inc., 1966.

14. Hermann, R.: Lie Groups for Physicists, New York: W.A. Benjamin, 1966.

15. Knapp,A.W., Stein, E. M.: Ann. Math. 93, 489 (1971).

16. Sciarrino, A., Toller, M.: J. Math. Phys. 8, 1252 (1967). Mukunda, N.: J. Math. Phys. 8, 2210 (1967); 9, 50 (1968). Ström, S.: Ark. Fys. 34, 215 (1967); 40, 1 (1969). Kuryan, J. G., Mukunda, N., Sudarshan,E.C.G.: J. Math. Phys. 9, 2100 (1968). Macfadyen, N.W.: J. Math. Phys. 12, 492 (1971).

17. Niederle, J.: J. Math. Phys. 8, 1921 (1968). Limić, N., Niederle,J.: Ann. Inst. Henri Poincaré IXA, 327 (1968).

18. Hannabuss, K. C.: Proc. Cambridge Phil. Soc. 70, 283 (1971).

J. Mickelsson

Department of Mathematics

University of Jyväskylä

Sammonkatu 6

Jyväskylä, Finland
J. Niederle

Institute of Physics of the

Czechoslovak Academy

of Sciences

Na Slovance 2

Prague, Czechoslovakia 